\title{
Some Philosophical Paradigms in Education of Modeling and Control
}

\author{
László Keviczky, Csilla Bányász" \\ Institute of Computer Science and Control, Hungarian Academy of Sciences, Hungary
}

Copyright $\bigcirc 2017$ by authors, all rights reserved. Authors agree that this article remains permanently open access under the terms of the Creative Commons Attribution License 4.0 International License

\begin{abstract}
The paper discusses some interesting, mainly philosophical paradigms of the modeling and control areas, which are still partly unsolved and/or only partially studied. First the possible introduction of a prejudice free control similar to the term for the modeling introduced by Rudi Kalman - is investigated. Next the real constraints in real control systems are discussed. It seems that these are the amplitude limit for the actuators in practical systems. Then the application of the HEISENBERG-type uncertainty relationship in control is discussed, combining the robustness of control and quality of modeling. Finally a special irregularity in the classical $L Q R$ control methodology is treated investing some unreachable poles in the anomaly. The paper constitutes a review of a few specific problems in the control theory. They are enumerated in keywords and discussed on the basis of the references. The authors aim at least to invite further authors to continue such a discussion.
\end{abstract}

Keywords Prejudice Free Control, Irregularities in Classical Control Methods, Real Constraints in Control Systems, the HEISENBERG Type Uncertainty Relation of Control

\section{Introduction}

Some scientists believe that everything has been solved in control, consequently nothing remained to study and/or research. The purpose of this paper is to recall some interesting philosophical paradigms in the areas of modeling and control to prove the contrary.

Only a few questions are discussed here, but there are many. Our aim is to encourage scientists to find further unsolved problems, blazes and interesting paradigms partly based on the modeling and control literature, partly on other disciplines.

If we can invite only a few further authors to continue our discussions then this effort is worth while.

In the sequel the YouLA parameterization [1], [2], [4], [5] will be used to discuss regulator and control system design. We found that this is very good basis for education, too.

The YoulA Parameterization

The YOULA- ( $Y$ or $Q$ ) -parameterization is a classical method for linear time invariant control system to characterize all realizable stabilizing regulators $(A R S)$ by

$$
C=\frac{Q}{1-Q P}
$$

for open-loop stable plant $P \in \mathrm{S}$, where $\mathrm{S}$ is the closed set of all stable proper real-rational systems, having all poles within the closed unit disc. The parameter"

$$
Q=\frac{C}{1+C P} ; Q \in \mathrm{S}
$$

ranges over all proper $(Q(\omega=\infty)$ is finite $)$, stable transfer functions [1], [5]. Observe that $Q$ is the transfer function from the $r$ to $u$ in the closed-loop (see Fig. 1), where $y_{\mathrm{n}}$ is the output disturbance (or noise) signal in a SISO (Single Input Single Output) system.

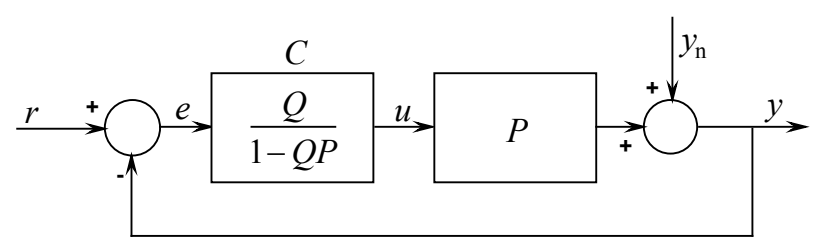

Figure 1. Closed-loop with an ARS regulator

The transfer characteristics of the closed-loop can be easily computed

$$
y=Q P r-(1-Q P) y_{\mathrm{n}}=y_{\mathrm{t}}+y_{\mathrm{d}}
$$

where $y_{\mathrm{t}}$ is the tracking (servo) and $y_{\mathrm{d}}$ is the regulating (or disturbance rejection) independent behaviors of the closed-loop response, respectively.

Because the $A R S$ regulator represented in Fig. 3 was formulated for an one-degree of freedom (1DF) control system, it is not surprising that the tracking part $y_{\mathrm{t}}$ of the 
transfer characteristics between $y$ and $r$ can not be set independently of the regulating behavior $y_{\mathrm{d}}$, i.e. independently of $Q$.

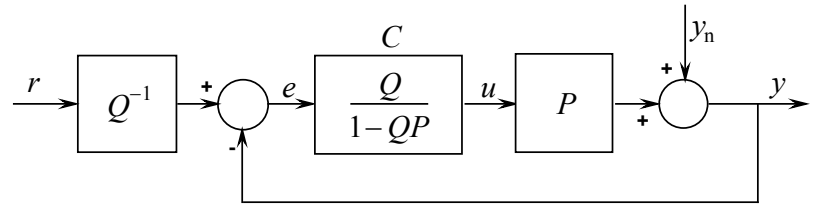

Figure 2. The modified control system with an ARS regulator opening the closed-loop

The Y-parameterization "almost" opens the closed-loop. Here "almost" means that $y_{\mathrm{t}}=Q P r$ is obtained instead of a real open-loop case with $y_{\mathrm{t}}=\operatorname{Pr}$. So we need a $Q^{-1}$ prefilter shown in Fig. 2, when the $A R S$ regulator really "virtually" opens the closed-loop as

$$
y=\operatorname{Pr}-(1-Q P) y_{\mathrm{n}}=y_{\mathrm{t}}+y_{\mathrm{d}}
$$

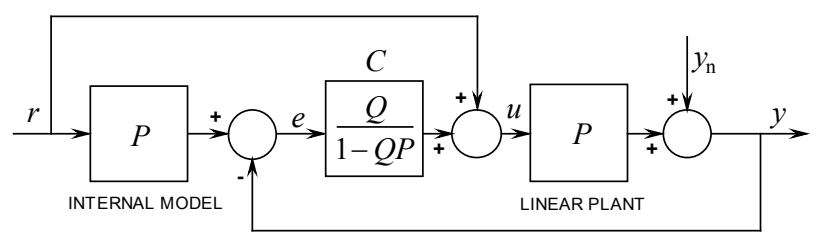

Figure 3. The $K$-B-parameterized $2 D F$ system with an $A R S$ regulator

An important and new observation of the authors was that the scheme in Fig. 2 is equivalent to the special control system given in Fig. 4 and its parameterization has been named as Keviczky-Bányász-(KB) parameterization [1], [2]. Since in the case of the special structure presented in Fig. 3 we have $y_{\mathrm{t}}=P r$, i.e., (3) holds, it is easy to introduce a new general form of any $2 D F$ control systems providing

$$
y=Q_{\mathrm{r}} P y_{\mathrm{r}}-(1-Q P) y_{\mathrm{n}}=y_{\mathrm{t}}+y_{\mathrm{d}}
$$

if a serial compensator $Q_{\mathrm{r}}$ is applied additionally as the Fig. 5 shows.

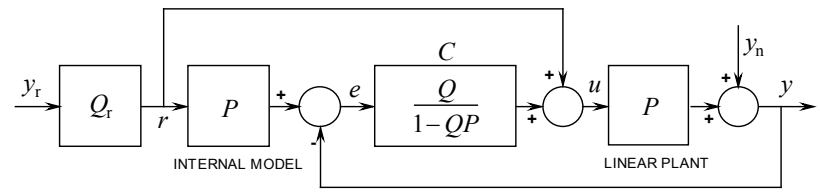

Figure 4. The general form of the $K$-B-parameterized $2 D F$ control system

Here and in the sequel the general notation $y_{\mathrm{r}}$ will be used for the reference signal for general $2 D F$ systems. Equation (5) shows that the tracking properties $y_{\mathrm{t}}=Q_{\mathrm{r}} P y_{\mathrm{r}}$ can independently be designed from the regulating behavior $y_{\mathrm{d}}=(1-Q P) y_{\mathrm{n}}$ by $Q_{\mathrm{r}}$.

The last scheme was later named as a generic two-degree of freedom $(G 2 D F)$ system [1], [2]. The $K-B$ parameterization for closed-loop control is not so widely known as the Youla-Kucera- $(Y-K)$ parameterization [4] however, it is much closer to a control engineering view and its most important advantage in $2 D F$ systems is that it virtually opens the closed-loop. However, this parameterization can only be applied for open-loop stable processes.

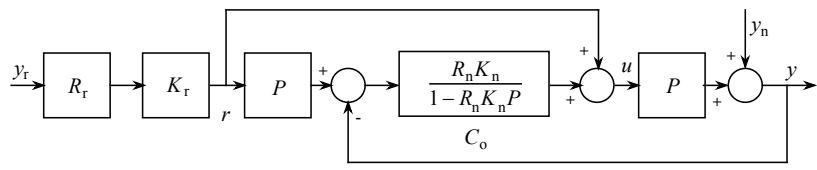

Figure 5. The generic $2 D F(G 2 D F)$ control system

A G2DF control system is shown in Fig. 5, where $y_{r}, u, y$ and $y_{\mathrm{n}}$ are the reference, process input, output and disturbance signals, respectively. The optimal discrete-time $A R S$ regulator of the $G 2 D F$ scheme [1], [2] is given by

$$
C_{\mathrm{o}}=\frac{R_{\mathrm{n}} K_{\mathrm{n}}}{1-R_{\mathrm{n}} K_{\mathrm{n}} P}=\frac{Q_{\mathrm{o}}}{1-Q_{\mathrm{o}} P}=\frac{R_{\mathrm{n}} G_{\mathrm{n}} P_{+}^{-1}}{1-R_{\mathrm{n}} G_{\mathrm{n}} P_{-} z^{-d}}
$$

where

$$
Q_{\mathrm{o}}=Q_{\mathrm{n}}=R_{\mathrm{n}} K_{\mathrm{n}}=R_{\mathrm{n}} G_{\mathrm{n}} P_{+}^{-1}
$$

is the associated $Y$-parameter [1], furthermore

$$
\begin{gathered}
Q_{\mathrm{r}}=R_{\mathrm{r}} K_{\mathrm{r}}=R_{\mathrm{r}} G_{\mathrm{r}} P_{+}^{-1} \\
K_{\mathrm{n}}=G_{\mathrm{n}} P_{+}^{-1} ; K_{\mathrm{r}}=G_{\mathrm{r}} P_{+}^{-1}
\end{gathered}
$$

assuming that the continuous-time process is factorable as

$$
P=P_{+} \bar{P}_{-}=P_{+} P_{-} e^{-s T_{\mathrm{d}}}
$$

and a discrete-time process is factorable as

$$
G=G_{+} \bar{G}_{-}=G_{+} G_{-} z^{-d}
$$

where $P_{+}, G_{+}$means the inverse stable $(I S)$ and $P_{-}, G_{-}$ the inverse unstable $(I U)$ factors, respectively. Here $T_{\mathrm{d}}$ is the continuous time delay and $z^{-d}$ corresponds to the discrete time delay, which is the integer multiple of the sampling time $T_{\mathrm{s}}$.

It was shown [1], [2] that the optimization of the $G 2 D F$ scheme can be performed in $\mathrm{H}_{2}$ and $\mathrm{H}_{\infty}$ norm spaces by the proper selection of the serial $K_{\mathrm{r}}$ and embedded $K_{\mathrm{n}}$ filters (compensators). These optimizations are reduced to the optimal computation of the $G_{\mathrm{r}}$ and $G_{\mathrm{n}}$ embedded filters. If $G_{\mathrm{r}}$ and $G_{\mathrm{n}}$ are optimally selected, then $C_{\mathbf{o}}$ denotes the optimal $A R S$ regulator in (6). It is interesting to see how the transfer characteristics of this system look like:

$$
\begin{aligned}
y & =R_{\mathrm{r}} K_{\mathrm{r}} P y_{\mathrm{r}}-\left(1-R_{\mathrm{n}} K_{\mathrm{n}} P\right) y_{\mathrm{n}}= \\
& =R_{\mathrm{r}} G_{\mathrm{r}} P_{-} e^{-s T_{\mathrm{d}}} y_{\mathrm{r}}-\left(1-R_{\mathrm{n}} G_{\mathrm{n}} P_{-} e^{-s T_{\mathrm{d}}}\right) y_{\mathrm{n}}=y_{\mathrm{t}}+y_{\mathrm{d}}
\end{aligned}
$$

or 


$$
\begin{aligned}
y & =R_{\mathrm{r}} K_{\mathrm{r}} G y_{\mathrm{r}}-\left(1-R_{\mathrm{n}} K_{\mathrm{n}} G\right) y_{\mathrm{n}}= \\
& =R_{\mathrm{r}} G_{\mathrm{r}} G_{-} z^{-d} y_{\mathrm{r}}-\left(1-R_{\mathrm{n}} G_{\mathrm{n}} G_{-} z^{-d}\right) y_{\mathrm{n}}=y_{\mathrm{t}}+y_{\mathrm{d}}
\end{aligned}
$$

Here $R_{\mathrm{r}}$ and $R_{\mathrm{n}}$ are stable and proper transfer functions, that are partly capable to place desired poles in the servo and the regulatory transfer functions, furthermore they are usually referred as reference signal and output disturbance predictors. They can even be called as reference models, so reasonably $R_{\mathrm{r}}(\omega=0)=1$ and $R_{\mathrm{n}}(\omega=0)=1$ are selected. In this case the obtained regulator is always an integrating one.

\section{Prejudice Free Control}

The knowledge of a process is never exact, independent of the method how its model is determined, whether measurement-based identification (ID) or

physico-chemical theoretical considerations are used. The uncertainty of the plant can be expressed by the absolute model error

$$
\Delta P=P-\hat{P}
$$

and the relative model error

$$
\ell=\frac{\Delta P}{\hat{P}}=\frac{P-\hat{P}}{\hat{P}}
$$

where $\hat{P}$ is the available nominal model used for regulator design and $P$ is the real plant.

The parameters of the plant may change in terms of their nominal values in a given range. The closed-loop control system needs to be stable under the given uncertainty ranges of the parameters.

Suppose that the open loop is stable. The regulator designed for the nominal plant ensures the stability of the nominal closed-loop control system. Let us analyze whether the system remains stable with the parameter uncertainties of the open loop. Stability is maintained if the NYQUIST diagram of the modified open loop does not encircle the $-1+0 j$ point.

If there is an uncertainty $\Delta P$ (or parameter change) in the transfer function of the plant, then if we apply the same regulator this uncertainty appears in the absolute error $\Delta L=C \Delta P$ of the loop transfer function, whereas its relative model error is

$$
\ell_{\mathrm{L}}=\frac{\Delta L}{\hat{L}}=\frac{L-\hat{L}}{\hat{L}}=\frac{C P-C \hat{P}}{C \hat{P}}=\frac{P-\hat{P}}{\hat{P}}=\ell
$$

Here $\hat{L}$ denotes the nominal and $L$ denotes the real loop transfer function.

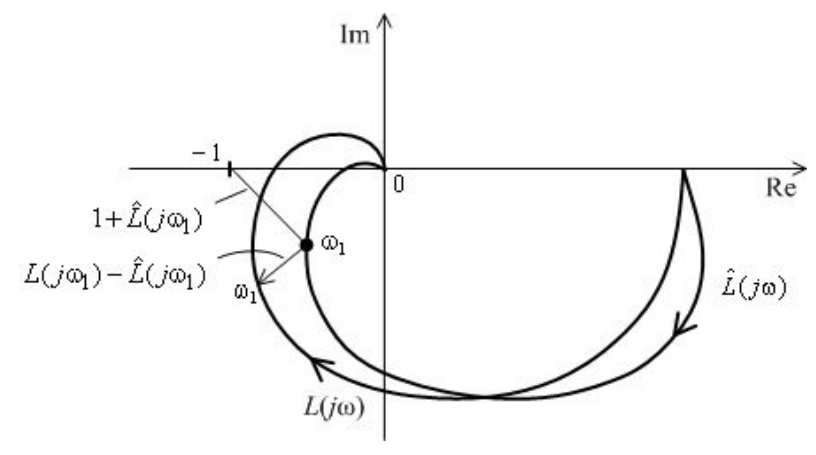

Figure 6. Change in the NYQUIST diagram of an uncertain system

Robust stability means that the closed-loop control system should not display unstable behavior even in the "worst case" parameter changes. The bound for $\Delta L$ can be formulated based in Fig. 6 by taking the simple geometrical considerations into account: the NYQUIST diagram will not encircle the $-1+0 j$ point, if the following relationship is satisfied for all frequencies:

$$
|\Delta L(j \omega)|=|\ell(j \omega)||\hat{L}(j \omega)|<|1+\hat{L}(j \omega)| \quad \forall \omega
$$

With further straightforward manipulations the necessary and sufficient condition for robust stability is obtained as

$$
|\ell(j \omega)|<\left|\frac{1+\hat{L}(j \omega)}{\hat{L}(j \omega)}\right|=\frac{1}{|\hat{T}(j \omega)|} \quad \forall \omega
$$

or

$$
|\hat{T}(j \omega)||\ell(j \omega)|<1 \quad \forall \omega
$$

where $\hat{T}=\hat{L} /(1+\hat{L})$ is the nominal complementary sensitivity function.

This form is also called the dialectic relationship of robust stability. In the design the first factor $|\hat{T}(j \omega)|$ is calculated for the supposed (known) nominal parameters of the plant, and thus it depends on the designer. The second factor 14 does not (or only partly) depends on the designer, as it contains the uncertainties in the knowledge of the plant or its unexpected parameter changes. In those frequency ranges where the uncertainty is large, unfortunately only small transfer gain can be designed for the closed-loop. Where $|\hat{T}(j \omega)|$ is high, very accurate information is needed to reach a small error. The higher the absolute value of the complementary sensitivity function, the smaller the permissible parameter uncertainty. 
The robust stability condition (4), (5) and (6) can be rearrange in the form of

$$
|C| \leq \eta\left|\hat{P}^{-1}\right|=\frac{1}{|1-| \ell||}\left|\hat{P}^{-1}\right|
$$

where the $\eta(|\ell|)$ function is plotted in Fig. 7. The interpretation of this function is very interesting. For small 14 modeling error a model based controller design is suggested, which usually based on the inverse of the nominal model $\hat{P}^{-1}$. For very large errors no regulator design is advised. However, in the midrange domain, where the error is around $100 \%$, the regulator design practically does not depend on our knowledge of the process. This area can be called "prejudice free" domain

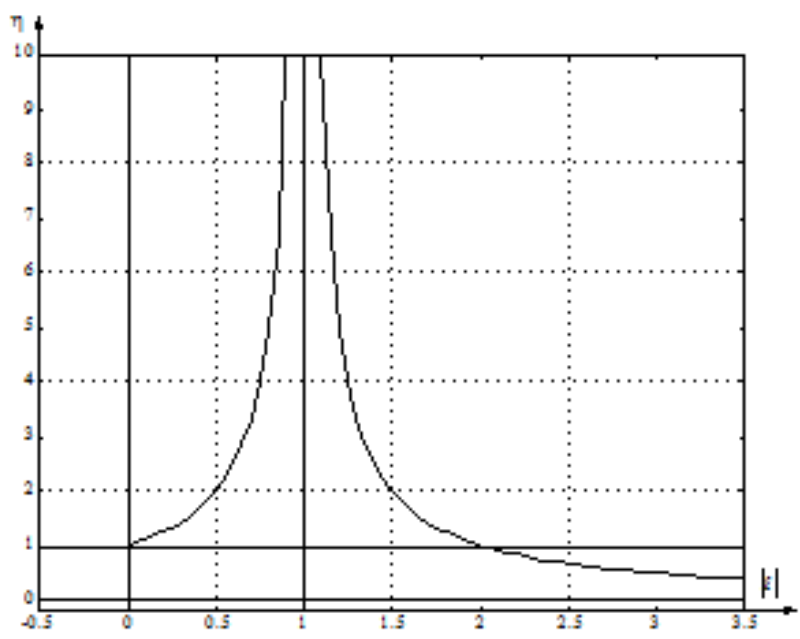

Figure 7. The $\eta$ in the function of $\ell$

Prejudice Free Control for Youla Parameterized Systems

The condition of robust stability for the $Y P$ control loops can be further simplified so the expression (18) becomes

$$
\begin{aligned}
|\hat{Q} \hat{P} \ell| & =\left|R_{\mathrm{n}} G_{\mathrm{n}} \hat{P}_{+}^{-1} \hat{P} \ell\right|=\left|R_{\mathrm{n}} G_{\mathrm{n}} \hat{P}_{-} e^{-s \hat{T}_{\mathrm{d}} \ell}\right|= \\
& =\left|R_{\mathrm{n}} G_{\mathrm{n}} \hat{P}_{-} \ell\right|=\left|R_{\mathrm{n}} G_{\mathrm{n}} \hat{P}_{-}\right||\ell|<1
\end{aligned}
$$

where $\hat{T}_{\mathrm{d}}$ is the dead time of the model and $\left|e^{-s \hat{T}_{\mathrm{d}}}\right|=1$.

The inequality (17), limiting the relative error, is now

$$
|\ell(j \omega)|<\frac{1}{\left|R_{\mathrm{n}}\right|\left|G_{\mathrm{n}} \hat{P}_{-}\right|} \quad \forall \omega
$$

If the process is $I S$, i.e., $\hat{P}_{-}=1$, then $G_{\mathrm{n}}=1$ can be chosen and the condition of robust stability can be further simplified as

$$
|(j \omega)\rangle<\frac{1}{\left|R_{\mathrm{n}}\right|} \quad \forall \omega
$$

i.e., it does not depend on the model $\hat{P}$ but only on the reference model or the design goal.

The reference model is an important parameter of the general YoulA design, by means of which the condition of robust stability (22) can be guaranteed. Let

$$
R_{\mathrm{n}}=\frac{1}{1+s T_{\mathrm{n}}}
$$

then the constraining condition of the right side of (22) can be seen in Fig. 8. Given the latter condition and choosing first-order reference model $R_{\mathrm{n}}$, we see that robust stability can be ensured even in the case of $100 \%$ relative model error. Furthermore for the high frequency domain a real prejudice free case is obtained,

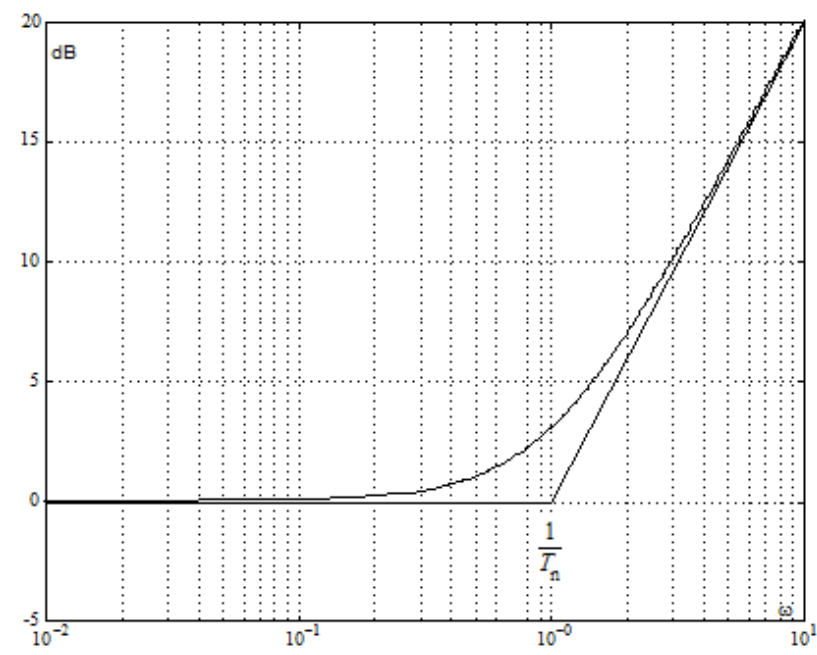

Figure 8. Condition constraining the relative model error in the case of the first-order reference model

If the process is $I U$, even the factor $\left|G_{\mathrm{n}} \hat{P}_{-}\right|$appears in (21), can significantly modify (22). Fig. 9 shows the case when two unstable zeros seriously decrease the prejudice free character of the stability. The worst case is when this factor has a large value in the region of the cut-off frequency.

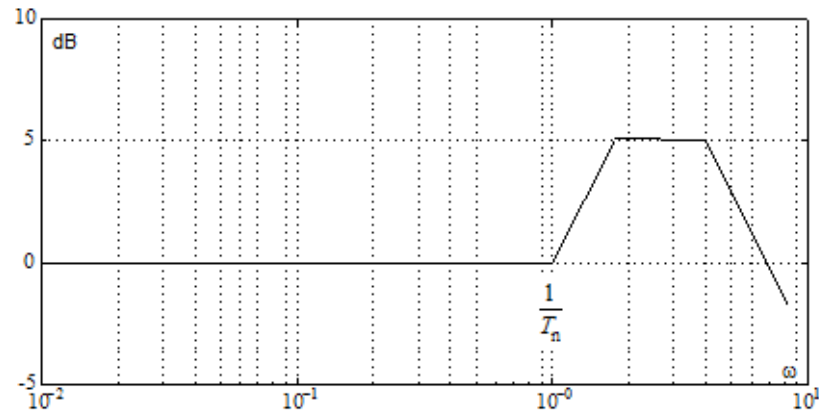

Figure 9. Conditions constraining the relative model error in the case of two unstable zeros

KALMAN was who tried to investigate the possibility of a 
prejudice free identification/modeling methodology [6]. He could not find any general applicable results, however many interesting, almost philosophical statements were developed.

\section{What are the Real Constraints in Control Systems}

\section{Process Behaviors Constraining the Control}

It was always an important question in the control theory papers what are the real constraints strongly effecting the result of the controller design.

We will discuss here the invariant process factors and the actuator operating signal domain here below. These factors are independent of the designer and of the available methods being either theoretically or experimentally founded.

One class of the constraints, which appear in the controller design is that the invariant factors of the real process, i.e., the unstable zeros and the time delay can not be eliminated with any control algorithm. So the best reachable closed-loop performance partly depends only on the process itself. If someone wants to change these elements only the redesign and rebuilt of the technology helps.

The ideal design goals formulated by the reference models $R_{\mathrm{r}}$ and $R_{\mathrm{n}}$ can be reached only for stable and inverse stable delay free processes. In case of inverse unstable processes only the approximate $R_{\mathrm{r}} G_{\mathrm{r}} P_{-} e^{-s T_{\mathrm{d}}}$ and $R_{\mathrm{n}} G_{\mathrm{n}} P_{-} e^{-s T_{\mathrm{d}}}$ goals can be reached [1]. For discrete time systems these goals are $R_{\mathrm{r}} G_{\mathrm{r}} G_{-} z^{-d}$ and $R_{\mathrm{n}} G_{\mathrm{n}} G_{-} z^{-d}$, respectively.

The influence of the unstable zeros can be somewhat attenuated using the $G_{\mathrm{r}}$ and $G_{\mathrm{n}}$ embedded filters. So only in case of stable process zeros we can obtain optimal controller independent of invariant process factor(s).

So before designing a control systems it is better to clarify what the process will allow us to reach and the final result will not depend on our skill or on the applied theoretical approach and/or methodology.

\section{Actuator Behaviors Constraining the Control}

There is another class of constraints which is independent on the applied regulator design and/or control systems applied. These are the always existing amplitude limits in the real actuators. Sometimes the theoretical control people forget about these constraints, because in their platforms, which is almost always computer technology, their life is limited only by the very big floating point number representations available at different software environments. Unfortunately the real actuators do not know these internal representation in spite of the fact that the modern control algorithms are based on discrete time computer control nowadays. The amplitude limit for a real actuator is always finite and we must not forget this reality. If we want to speed up a slow process by modern control, the result does not depends on the theoretical strength of the applied algorithm, it depend primarily on the available amplitude limit which can be applied at the output of the actuator.

The regulators always invoke zeros to accelerate the process. To demonstrate this let us investigate the case shown in Fig. 10, where a phase-lead element is connected in serial before the first-order lag element. In the first moment, a signal value of 10 appears at the output of the phase-lead element and at the input of the first-order lag element for the effect of a unit step signal. The first-order lag starts with a high gradient in order to reach this value as soon as possible with its time constant and by the time the input signal is settling down the output has almost reached its steady-state value. The cost of the acceleration is the so-called over-excitation, i.e., the ratio of the initial and final signal values at the input of the lag. The acceleration can be reached only by over-excitation greater than one. In many cases, it is worth applying pole cancellation, when zeros are invoked to cancel the undesirable poles which cause slow operation, and instead a pole ensuring more favorable behavior is inserted.

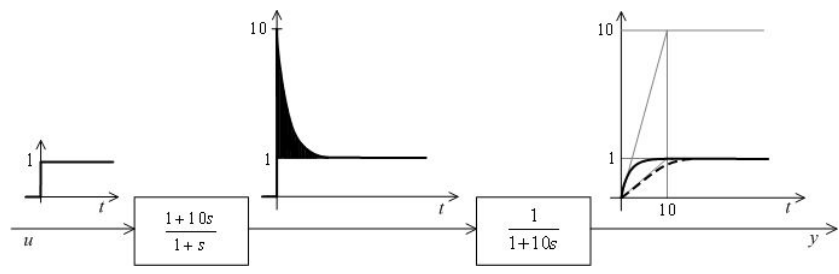

Figure 10. Insertion of a zero may accelerate the system at the cost of over-excitation

Thus it is obvious that the over-excitation means the control equipments will have an initial peak at their output as a response to unit step commands or disturbances. The problem is that the output of the regulators in the closed-loop control, or the output of the actuators gaining the signal for the proper level are always amplitude-restricted.

$$
|u(t)| \leq U_{\max }
$$

The sensitivity function of the real closed-loop can be written in the following decomposed form:

$$
\begin{aligned}
& S=\underbrace{\left(1-R_{\mathrm{n}}\right)}_{S_{\text {des }}}+\underbrace{S_{\text {perf }}}_{\underbrace{\left(R_{\mathrm{n}}-\hat{T}\right)}_{\text {real }}}-\underbrace{(T-1)}_{S_{\text {mod }}(T-\hat{T})}=S_{\text {des }}+S_{\text {real }}+S_{\text {mod }}= \\
& =\underbrace{\left(1-R_{\mathrm{n}}\right)}_{S_{\text {des }}}+\underbrace{\left(R_{\mathrm{n}}-T\right)}_{S_{\text {perf }}}=S_{\text {des }}+S_{\text {perf }}=\underbrace{(1-\hat{T})}_{S_{\text {contr }}}+S_{\text {mod }}= \\
& =\underbrace{(1-\hat{T})}_{S_{\text {des }}+S_{\text {real }}}+S_{\text {mod }}=S_{\text {contr }}+S_{\text {mod }}
\end{aligned}
$$


Here $S_{\text {des }}=\left(1-R_{\mathrm{n}}\right)$ is the design loss, $S_{\text {real }}=\left(R_{\mathrm{n}}-\hat{T}\right) \quad$ is the realizability loss, $S_{\text {mod }}=-(T-\hat{T})=\hat{T}-T$ refers to the modeling loss in the sensitivity function. In its other form $S_{\text {contr }}=(1-\hat{T})$ means the decomposed term referring to the control loss, $S_{\text {perf }}=\left(R_{\mathrm{n}}-T\right)$ to the performance loss. Each term can be interpreted and explained very easily.

The above triple decomposition of the sensitivity functions gives a good insight into the limit-optimality (limits of the optimality) of closed-loop control systems, i.e., the characterization of the best control achievable. As regards this distinction optimality criteria need to be created for each term, i.e.,

$$
\begin{gathered}
J_{\text {dist }} \leq J_{\text {des }}^{\mathrm{n}}+J_{\text {real }}^{\mathrm{n}}+J_{\text {mod }}^{\mathrm{n}}=\left\|S_{\text {des }}\right\|+\left\|S_{\text {real }}\right\|+\left\|S_{\text {mod }}\right\| \\
J_{\text {track }} \leq J_{\text {des }}^{\mathrm{r}}+J_{\text {real }}^{\mathrm{r}}+J_{\text {mod }}^{\mathrm{r}}=\left\|S_{\text {des }}^{\mathrm{r}}\right\|+\left\|S_{\text {real }}^{\mathrm{r}}\right\|+\left\|S_{\text {mod }}^{\mathrm{r}}\right\|
\end{gathered}
$$

both for the tracking and disturbance rejection behaviors. Here the notation $\| \mathbb{\|}$ is used to express the optimality criterion. In strict mathematical analysis this notation is used to refer to the chosen norm of the transfer function. It is not an easy task to optimize all three terms simultaneously. In practice iterative techniques are used, whereby the optimization problem is solved step-by-step.

The optimization of the first term in the decomposition of the sensitivity function (25) means the determination of the best (fastest) reachable reference models $R_{\mathrm{r}}=R_{\mathrm{r}}^{\mathrm{opt}}$ and $R_{\mathrm{n}}=R_{\mathrm{n}}^{\text {opt }}$, i.e., the solution of the task under the constraints

$$
\begin{gathered}
R_{\mathrm{r}}^{\mathrm{opt}}=\arg \left\{\left.\min _{R_{\mathrm{r}}}\left(J_{\text {des }}^{\mathrm{r}}\right)\right|_{u \in \boldsymbol{U}}\right\}=\arg \left\{\min _{R_{\mathrm{r}}}\left\|1-R_{\mathrm{r}}\right\|_{u \in \boldsymbol{U}}\right\} \\
R_{\mathrm{n}}^{\mathrm{opt}}=\arg \left\{\left.\min _{R_{\mathrm{n}}}\left(J_{\mathrm{des}}^{\mathrm{n}}\right)\right|_{u \in \boldsymbol{U}}\right\}=\arg \left\{\min _{R_{\mathrm{n}}}\left\|1-R_{\mathrm{n}}\right\|_{u \in \boldsymbol{U}}\right\}
\end{gathered}
$$

where the chosen criteria $J_{\text {des }}^{\mathrm{r}}=\left\|1-R_{\mathrm{r}}\right\| \quad$ and $J_{\text {des }}^{\mathrm{n}}=\left\|1-R_{\mathrm{n}}\right\|$ state that each reference model should approach the ideal unity. This task must be solved under the constraints for the regulator output $u \in U$. Here $U$ means the allowable region for $u$, i.e., $U:|u| \leq 1$ (see (24)).

\section{Redesign of the Reference Model}

The optimization task (27) is very difficult because the solution is always on the border of the limited region. There is no analytical solution except for some low-order simple cases. The optimal reference models are usually determined by simulation (CAD tools). Note that under the given constraints faster reference models cannot be used to solve the task (27). Vice versa, if under the constraints and design goal there is no solution for the reference models then the only option is to prescribe a less demanding (usually slower) model. Thus the best (fastest) reachable response of the closed system basically depends on the constraints of the control output. Of course, equation (27) contains the applied regulator and also the process in a complex way; thus it is a closed-loop. Therefore the optimality of the regulator depends on the process, the model and the invariant factors.

Even in the case of a very careful design it can happen that the over-actuated output of the obtained regulator is beyond the acceptable signal domain. Then the original design goals need to be reduced. The advantage of the $K B$ parameterization of generic $2 D F$ control loops is that only the problematic (over-demanding) reference models $R_{\mathrm{r}}$ and $R_{\mathrm{n}}$ need to be changed to accommodate less demanding design conditions. Usually this can be performed only step by step via an iterative procedure. The steps can contain model simulation and also experiments on the real process. Therefore the optimization is often termed the redesign of the reference model. In the case of low-order reference models the time constant of the model (i.e., the bandwidth) can be determined by explicit design expression if the process model and the amplitude constraint $U_{\text {max }}$ are known.

Let the process be given by the pulse transfer function

$$
G=\frac{0.125 z^{-1}\left(1+1.6 z^{-1}\right)}{\left(1-0.5 z^{-1}\right)\left(1-0.8 z^{-1}\right)}
$$

Apply a combined iterative identification and control test [12]. The following reference models of unity gain are used for the design

$$
R_{\mathrm{r}}=\frac{0.9 z^{-1}}{1-0.1 z^{-1}} \text { and } R_{\mathrm{r}}=\frac{0.9 z^{-1}}{1-0.1 z^{-1}}
$$

At the start of the iteration the model

$$
\hat{G}_{\mathrm{o}}=\frac{0.1 z^{-1}\left(1+4.0 z^{-1}\right)}{\left(1-0.2 z^{-1}\right)\left(1-0.9 z^{-1}\right)}
$$

is assumed. A square signal with periodic time of 40 samples is applied as reference signal. In the simulation it is assumed that the additive noise $y_{\mathrm{n}}$ is white noise, whose variance is $\sigma_{y_{\mathrm{n}}}=0.01$. The number of the processed samples is $N=100$. Because of the small output noise the identification is performed by a simple off-line $L S$ method. The regulator is designed by the $Y P$ method, assuming an $I U$ process. 


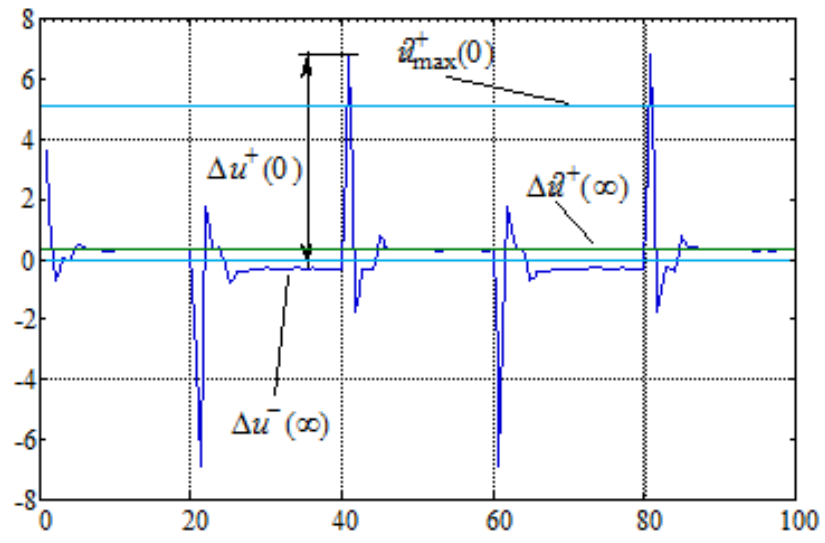

Figure 11. Response of the $Y P$ regulator before the iteration

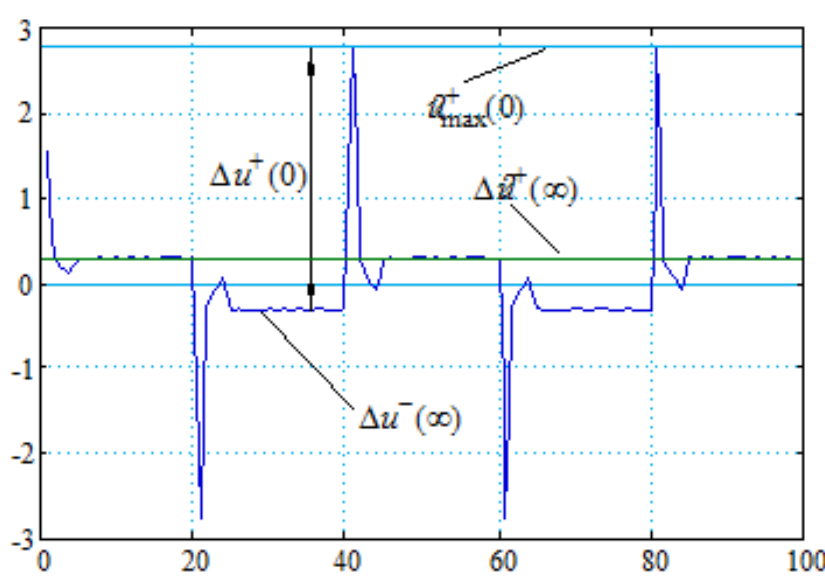

Figure 12. Response of the $Y P$ regulator after the iteration

The output of the regulator is presented in Fig. 11, where it is seen that the over-excitation is very high at $900 \%$, i.e., $u_{\mathrm{t}}=9$. Assume that the actuator can realize only $\bar{u}_{\mathrm{t}}=5$. This requires the redesign of the reference model $R_{\mathrm{r}}$.

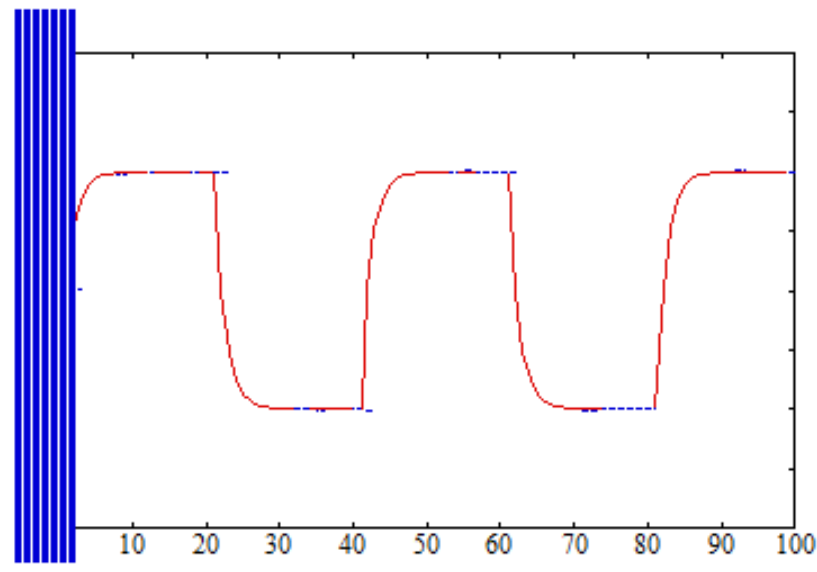

Figure 13. Output of the reference model and the process

The condition $u_{\mathrm{t}} \leq \bar{u}_{\mathrm{t}}=5$ needs to be prescribed for the reference model redesign iteration. It can be seen in Fig. 12 that the control output is according to the prescribed over actuation by the end of the iteration. The obtained redesigned reference model is

$$
\bar{R}_{\mathrm{r}}=\frac{0.5022 z^{-1}}{1-0.4978 z^{-1}}
$$

Figure 13 shows the time function of the output of the reference model (continuous line) and the closed system (dashed line). Fig. 14 presents the BoDE diagram of the original (continuous line) and the redesigned (dashed line) reference model. The prescribed over-excitation could be reached only by substantially slowing down the closed system.

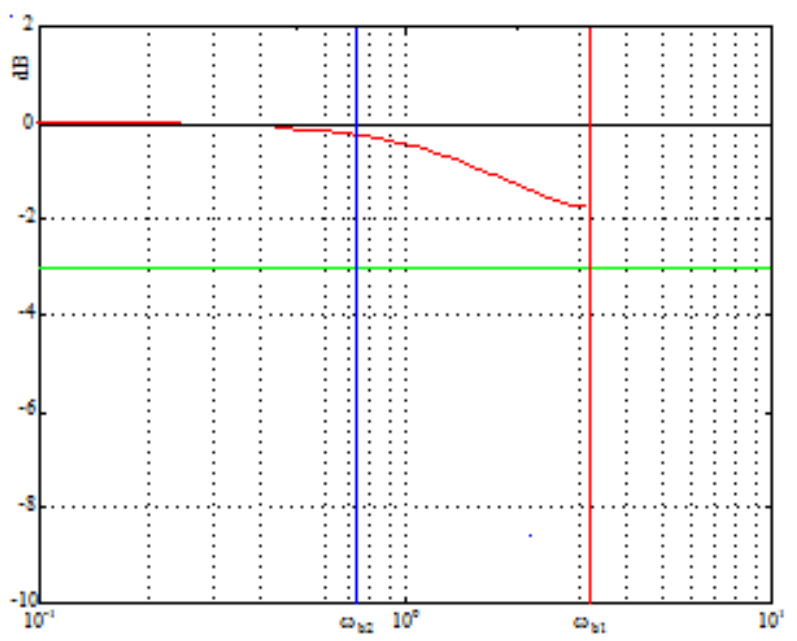

Figure 14. BoDE diagram of the original and the new reference model

The final conclusion is that the control quality can never be prejudice free, more exactly never process independent and never the installed actuator independent property of the final control system.
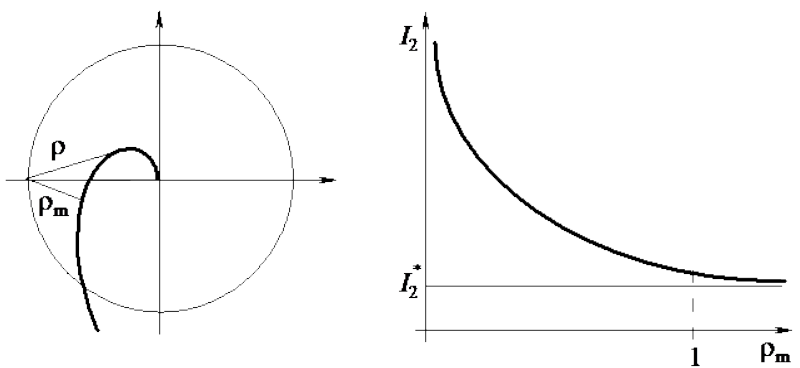

$\rho_{\mathrm{m}}(\ddot{e})=\min _{\omega}\{\rho(\omega, \ddot{e})\}=\min _{\omega}(\rho)$ $\frac{I_{2}^{*}}{I_{2}} \rho_{\mathbf{m}} \leq$ limit

Figure 15. Illustration of the inequality of (9.5.1)

\section{The Heisenberg-Type Uncertainty of Control}

The condition of robust stability (18) already contains a product inequality. Here $|\hat{T}(j \omega)|$ (although it is usually called a design factor) can be considered as the quality 
factor of the control. The other factor, however, can be considered as the relative correctness of the applied model. In the light of practical experience control engineers favor applying a mostly heuristic expression

(quality of the control) $\times$ (robustness of the control) $\leq$ limit

This product inequality can be simply demonstrated by the integral criteria of classical control engineering. Let $I_{2}$

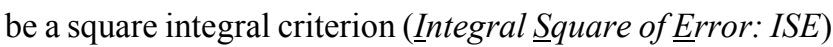
whose optimum is $I_{2}^{*}$ when the regulator is properly set, and the NYQUIST stability limit (i.e., robustness measure) is $\rho_{\mathrm{m}}$. The well-known empirical, heuristics formula is

$$
\frac{I_{2}^{*}}{I_{2}} \rho_{\mathrm{m}} \leq \text { limit }
$$

The inequality is illustrated in Fig. 15. The fact that the quality of the identification (which is the inverse of the model correctness) can have a certain relationship with the robustness of the control, is not very trivial. This can be observed only in a special case, namely in the identification technique based on $K B$ parameterization [1] [2] when $\xi_{\mathbb{D}}=-\varepsilon \%$. Introduce a new relationship for the characterization of the quality of the control

$$
\delta=\delta(\omega, \hat{C})=\frac{|-\tilde{e}(j \omega)|}{\left|y_{\mathrm{n}}(j \omega)\right|}=\left|\frac{1}{1+\hat{C} P}\right|=\frac{1}{|1+\tilde{L}|}
$$

Notice that $\delta$ is the absolute value of the sensitivity function. Obviously, $\delta \rho=1$ for all frequencies (here $\rho=|1+\tilde{L}|$ ). Of course, the same equalities are valid for the minimum and maximum values, i.e.,

$$
\begin{aligned}
& \rho_{\mathrm{m}}(\hat{C})=\min _{\omega}[\rho(\omega, \hat{C})]=\min _{\omega}(\rho) \\
& \delta_{\mathrm{M}}(\hat{C})=\max _{\omega}[\delta(\omega, \hat{C})]=\max _{\omega}(\delta)
\end{aligned} \text {; i.e., their }
$$

product is unity: $\delta_{\mathrm{M}} \rho_{\mathrm{m}}=1$

Denote the worst value of these measures by

$$
\begin{gathered}
\widehat{\rho}_{\mathrm{m}}=\max _{\hat{C}}\left\{\min _{\omega}[\rho(\omega, \hat{C})]\right\}=\max _{\hat{C}}\left[\min _{\omega}(\rho)\right] \\
\breve{\delta}_{\mathrm{M}}=\min _{\hat{C}}\left\{\max _{\omega}[\delta(\omega, \hat{C})]\right\}=\min _{\hat{C}}\left[\max _{\omega}(\delta)\right] \\
\breve{\delta}_{\mathrm{M}} \hat{\rho}_{\mathrm{m}}=1
\end{gathered}
$$

The above three basic relationships can be summarized in the inequalities below

$$
\delta \rho=1 ; \delta_{\mathrm{M}} \rho_{\mathrm{m}}=1 ; \breve{\delta}_{\mathrm{M}} \hat{\rho}_{\mathrm{m}}=1
$$

where the following simple calculations prove the existence of (34) and (35)

$$
\begin{aligned}
\rho_{\mathrm{m}}(\hat{C}) & =\min _{\omega}|1+\hat{C} P|=\frac{1}{\max _{\omega}\left|\frac{1}{1+\hat{C} P}\right|}=\frac{1}{\left\|\frac{1}{1+\hat{C} P}\right\|_{\infty}}= \\
& =1 / \delta_{\mathrm{M}}(\hat{C}) \\
\breve{\delta}_{\mathrm{M}} & =\min _{\hat{C}}\left\{\max _{\omega}\left|\frac{1}{1+\hat{C} P}\right|\right\}=\frac{1}{\max _{\hat{C}}\left\{\max _{\omega}\left|\frac{1}{1+\hat{C} P}\right|\right\}} \\
& =1 / \max _{\hat{C}}\left\{\min _{\omega}|1+\hat{C} P|\right\}=1 / \hat{\rho}_{\mathrm{m}}
\end{aligned}
$$

Given (34), (35) and (36) further basic, almost trivial, inequalities can also be simply formulated

$$
\begin{aligned}
& \breve{\delta}_{\mathrm{M}} \leq \delta_{\mathrm{M}}(\hat{C}) ; \rho_{\mathrm{m}}(\hat{C}) \leq \widehat{\rho}_{\mathrm{m}} \\
& \frac{1}{\widehat{\rho}_{\mathrm{m}}}=\breve{\delta}_{\mathrm{M}} \leq \delta_{\mathrm{M}}(\hat{C})=\frac{1}{\rho_{\mathrm{m}}(\hat{C})} \\
& \frac{1}{\delta_{\mathrm{M}}(\hat{C})}=\rho_{\mathrm{m}}(\hat{C}) \leq \bar{\rho}_{\mathrm{m}}=\frac{1}{\breve{\delta}_{\mathrm{M}}}
\end{aligned}
$$

The above results are not surprising. The fact, that they are valid even for the modeling error in the case of $K B$-parameterized identification methods makes them special. So it can be clearly seen that when the modeling error decreases, the robustness of the control increases. Namely, if the minimum of the modeling error $\breve{\delta}_{\mathrm{M}}$ is decreased, then the maximum of the minimum robustness measure $\widehat{\rho}_{\mathrm{m}}$ is increased, since $\breve{\delta}_{\mathrm{M}} \hat{\rho}_{\mathrm{m}}=1$.

Similar relationships can be obtained if the $\mathcal{H}_{2}$ norm of the "joint" modeling and control error is used instead of the absolute values. Introduce the following relative fidelity measure

$$
\sigma=\frac{\left\|\varepsilon_{\mathrm{ID}}\right\|_{2}}{\left\|y_{\mathrm{n}}\right\|_{2}}=\frac{\|\tilde{e}\|_{2}}{\left\|y_{\mathrm{n}}\right\|_{2}} ;\left\|y_{\mathrm{n}}\right\|_{2} \neq 0
$$

The upper limit for this measure can be formulated as

$$
\sigma=\frac{\left\|\varepsilon_{\mathrm{ID}}\right\|_{2}}{\left\|y_{\mathrm{n}}\right\|_{2}}=\frac{\|\tilde{e}\|_{2}}{\left\|y_{\mathrm{n}}\right\|_{2}} \leq\left\|\frac{1}{1+\hat{C} P}\right\|=\delta_{\mathrm{M}}(\hat{C})
$$

so it is very easy to find similar equations for $\sigma$. Let

$$
\begin{gathered}
\sigma_{\mathrm{M}}(\hat{C})=\max _{\ell}[\sigma(\ell, \hat{C})] \text { and } \\
\breve{\sigma}_{\mathrm{M}}=\min _{\hat{C}}\left\{\max _{\ell}[\sigma(\ell, \hat{C})]\right\} .
\end{gathered}
$$

Using these definitions and the former equations we obtain the following interesting relationship

$$
\breve{\sigma}_{\mathrm{M}} \leq \breve{\sigma}_{\mathrm{M}}(\hat{C}) \leq \delta_{\mathrm{M}}(\hat{C})=\frac{1}{\rho_{\mathrm{m}}(\hat{C})}
$$

for the relative quadratic identification error. 
Use the first-order reference model

$$
R_{\mathrm{n}}=\frac{b_{\mathrm{n} 1} z^{-1}}{1+a_{\mathrm{n} 1} z^{-1}}
$$

for the design of the noise rejection in the $I S$ process. Here the maximum of the robustness measure is $\hat{\rho}_{\mathrm{m}}^{\mathrm{o}}=\hat{\rho}_{\mathrm{m}, \mathrm{IS}}^{\mathrm{o}}=0.9$ according to

$$
\hat{\rho}_{\mathrm{m}, \mathrm{IS}}^{\mathrm{o}}=\widehat{\rho}_{\mathrm{m}, \mathrm{IS}}(\ell=0)=\frac{1}{\left\|1-R_{\mathrm{n}}\right\|_{\infty}}=\min _{\omega}\left|\frac{1}{1-R_{\mathrm{n}}}\right|
$$

and

$$
\widehat{\rho}_{\mathrm{m}, \mathrm{IS}}^{\mathrm{o}}=\frac{\left|a_{\mathrm{n} 1}-1\right|}{2}
$$

This gives a robust measure of $\hat{\rho}_{\mathrm{m}, \mathrm{IS}}^{\mathrm{o}}=0.9$ under the parameters $b_{\mathrm{n} 1}=0.2$ and $a_{\mathrm{n} 1}=-0.8$ chosen for reference models with unity gain (same as in (29)).

The values of the typical variables (see above) are

$$
\begin{gathered}
\breve{\delta}_{\mathrm{M}}^{\mathrm{o}}=\frac{1}{\widehat{\rho}_{\mathrm{m}}^{\mathrm{o}}}=\frac{2}{\left|a_{\mathrm{n} 1}-1\right|}=1.1111 \Rightarrow \breve{\delta}_{\mathrm{M}}^{\mathrm{o}} \widehat{\rho}_{\mathrm{m}}^{\mathrm{o}}=1 \\
\sigma_{\mathrm{M}}^{\mathrm{o}}=\frac{1}{\sqrt{\hat{\rho}_{\mathrm{m}}^{\mathrm{o}}}}=\sqrt{\frac{2}{\left|a_{\mathrm{n} 1}-1\right|}}=1.054 \Rightarrow \\
\sigma_{\mathrm{M}}^{\mathrm{o}} \hat{\rho}_{\mathrm{m}}^{\mathrm{o}}=0.9486 \leq \breve{\delta}_{\mathrm{M}}^{\mathrm{o}} \hat{\rho}_{\mathrm{m}}^{\mathrm{o}}=1
\end{gathered}
$$

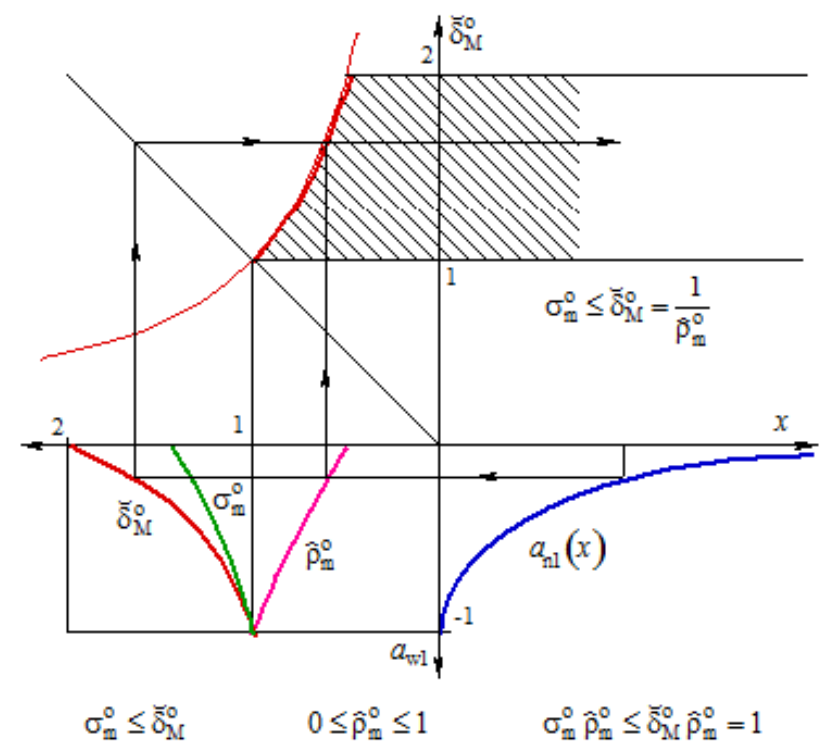

Figure 16. Illustration of uncertainty relationships (41)

Considering the data of (46) and applying again the relative sampling time $x=T_{\mathrm{s}} / T_{\mathrm{n}}$, the different measures in (42) are illustrated in Fig. 16. Here $T_{\mathrm{n}}$ is the time constant of the continuous time (CT) first-order reference model.
Introduce the following coefficient for the excitation caused by the reference signal

$$
\xi=\frac{\left\|y_{\mathrm{r}}\right\|_{2}}{\left\|\mathrm{y}_{\mathrm{n}}\right\|_{2}} ;\left\|\mathrm{y}_{\mathrm{n}}\right\|_{2} \neq 0
$$

which represents a signal/noise ratio. Investigate the product $\sigma \rho$ (which is called the uncertainty product) in an iterative procedure where the relative error $\mathbb{E}$ of the model is improved gradually. For simplicity, let us assume an IS process. It can be simply derived that

$$
\begin{aligned}
\sigma \rho_{\mathrm{m}} & \leq \sigma \bar{\rho}_{\mathrm{m}}^{\mathrm{o}} \leq \frac{1+\xi\left\|R_{\mathrm{n}} \ell\right\|_{\infty}}{\min \left|1+R_{\mathrm{n}} \ell\right|} \leq\left.\frac{1+\xi\left\|R_{\mathrm{n}}\right\|_{\infty}\|\ell\|_{\infty}}{1-\xi\left\|R_{\mathrm{n}}\right\|_{\infty}\|\ell\|_{\infty}}\right|_{\ell \rightarrow 0}= \\
& =\sigma_{\mathrm{o}} \widehat{\rho}_{\mathrm{m}}^{\mathrm{o}}=1
\end{aligned}
$$

i.e.,

$$
\begin{gathered}
\sigma \rho_{\mathrm{m}} \leq \sigma \widehat{\rho}_{\mathrm{m}}^{\mathrm{o}} \underset{\ell \rightarrow 0}{\leq} \sigma_{\mathrm{o}} \rho_{\mathrm{m}}^{\mathrm{o}}=1 \text { or } \\
\sigma \leq \sigma_{\mathrm{o}}=\frac{1}{\hat{\rho}_{\mathrm{m}}^{\mathrm{o}}}=\breve{\delta}_{\mathrm{M}}^{\mathrm{o}} \leq \delta_{\mathrm{M}}=\frac{1}{\rho}
\end{gathered}
$$

where $\sigma_{\mathrm{o}}=\sigma(\ell=0)$. Similarly to the notations $\sigma_{\mathrm{M}}(\hat{C})$ and $\bar{\sigma}_{\mathrm{M}}$ applied above, the notations $\sigma_{\mathrm{m}}(\ell)=\min _{\ell}[\sigma(\ell, \hat{C})]$ and $\sigma_{\mathrm{m}}^{\mathrm{o}}=\sigma_{\mathrm{m}}(\ell=0)$ can also be introduced. It is not an easy task, however, to derive the relationship between $\sigma_{\mathrm{m}}^{\mathrm{o}}$ and $\sigma_{\mathrm{o}}$ or $\bar{\sigma}_{\mathrm{M}}$ and $\sigma_{\mathrm{M}}(\hat{C})$. The simplest case to investigate (50) is when $\ell=0$, since then

$$
\sigma_{\mathrm{m}}^{\mathrm{o}} \leq \sigma_{\mathrm{M}}^{\mathrm{o}}(\hat{C}) \leq \delta_{\mathrm{M}}^{\mathrm{o}}(\hat{C})=\frac{1}{\rho_{\mathrm{m}}^{\mathrm{o}}(\hat{C})}
$$

This equation gives a new uncertainty relationship, according to which

$$
\frac{\|\tilde{e}\|_{2}}{\left\|y_{\mathrm{n}}\right\|_{2}} \min _{\hat{C}}|1+\hat{C} P| \underset{\ell \rightarrow 0}{\leq} 1
$$

The product of the modeling accuracy and the robustness measure of the control must not be greater than one, when the optimality condition $\ell=0$ is reached. The obtained uncertainty relation can be written in another form, since

$$
\sup \left\{\frac{\|\tilde{e}\|_{2}}{\left\|y_{\mathrm{n}}\right\|_{2}}\right\} \min _{\hat{C}}|1+\hat{C} P|_{\ell=0}=1
$$

The earlier results of control engineering referred only for the statement that the quality of the control cannot be improved, only at the expense of the robustness, so this result, which connects the quality of the identification and the robustness of the control, can be considered novel.

This phenomenon can arguably be considered as the HEISENBERG-type uncertainty relationship of control 
engineering, according to which

$$
\frac{1}{\Delta z} \frac{1}{\Delta p} \leq 1
$$

Here $\Delta z$ and $\Delta p$ are the alterations of the canonical coordinate and the impulse variables, respectively, and thus their inverse corresponds to the generalized accuracy and "rigidity" which are known as performance and robustness in control engineering.

The consequence of the new uncertainty relation is very simple: $K B$-parameterized identification is the only method where the improvement of the modeling error also increases the robustness of the control. With other methods, and other identification topology, modeling and control errors are interrelated in a very complex way, and in many cases this relation cannot be given in an explicit form. This is the main reason why it is difficult to elaborate a method which guarantees, or at least forces, similar behavior by the two errors, though some results can be found in [3].

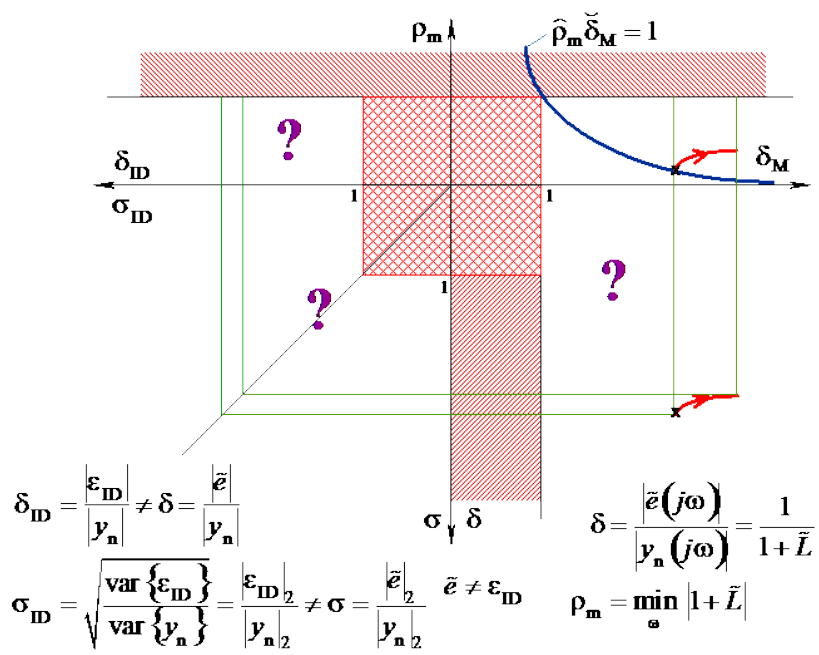

Figure 17. Relationship between the control and identification error in the general case

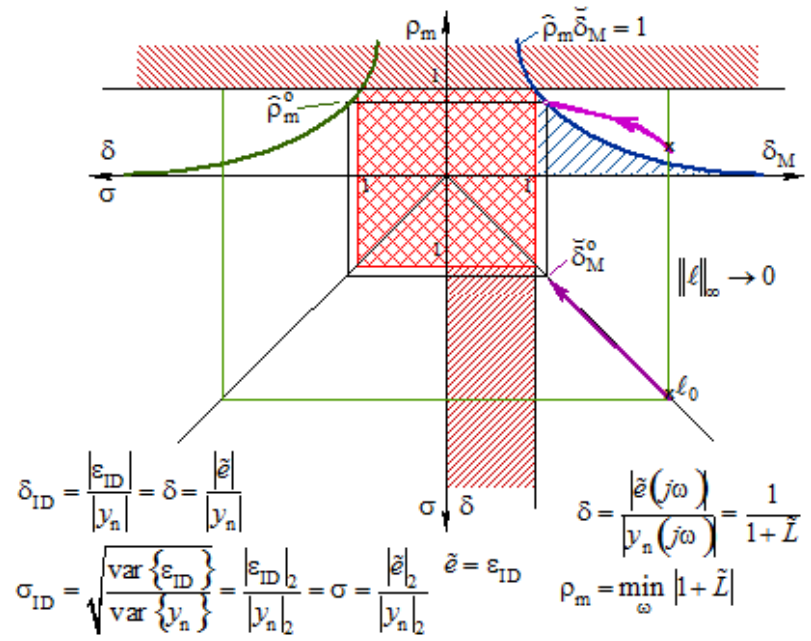

Figure 18. Relationship between the control and identification error in the case of the $K B$-parameterized identification method

There is a myth in the literature concerning the antagonistic conflict between control and identification. A "good" regulator minimizes the internal signal changes in the closed loop and therefore most of the identification methods, which use these inner signals provide worse modeling error, if the regulator is better. The exciting signal of $K B$-parameterized identification is an outer signal and therefore the phenomenon does not exist. The relevant feature of this relationship is shown in Figs. 17 and 18 for a general identification method and a $K B$-parameterized technique.

In Fig. 17, there is no clear relation between $\delta_{\text {ID }}$ and $\delta$, or $\sigma_{\mathrm{ID}}$ and $\sigma$, and therefore there it is not guaranteed that minimizing $\delta_{\mathrm{M}}$ increases $\rho_{\mathrm{m}}$. In Fig. $18 \delta_{\mathrm{ID}}=\delta$ and $\sigma_{\mathrm{ID}}=\sigma$, and thus the minimization of $\delta_{\mathrm{M}}$ directly maximizes $\rho_{\mathrm{m}}$. Thus if during the iterative identification the condition $\left\|\ell_{k}\right\|_{\infty} \underset{k \rightarrow \infty}{=0} 0$ is guaranteed then, at the same time, the convergences $\breve{\delta}_{\mathrm{M}}^{k} \underset{k \rightarrow \infty}{=} \breve{\delta}_{\mathrm{M}}^{\mathrm{o}}$ and $\hat{\rho}_{\mathrm{m}}^{k} \underset{k \rightarrow \infty}{=} \widehat{\rho}_{\mathrm{m}}^{\mathrm{o}}$ are ensured.

\section{Irregularities in Classical Control Methods}

A further myth in the control literature is that everything is right and errorless in the classical works of theory. This is unfortunately (or fortunately ?) is not right. T. Keviczky recognized that the solution of the classical $L Q R$ paradigm does not provide full pole placement and some areas can be considered as irregularities of this classical theory. Keviczky and Bányász [9], [10] gave a detailed analysis proving that there are some poles, it is interesting that the slower poles, which can not be allocated by the classical methodology. In some earlier references it was also studied that some poles are unreachable in the $L Q R$ theory by Johnson [11]. Finally Bokor and Keviczky [12], [13] presented a possible method, which solves the irregularity of this paradigm.

The LQR (Linear system - Quadratic criterion Regulator) problem

This optimization paradigm was formulated by the general, quadratic criterion [7], [8]

$$
I=\frac{1}{2} \int_{0}^{\infty}\left[\boldsymbol{x}^{\mathrm{T}}(t) \boldsymbol{W}_{\mathrm{x}} \boldsymbol{x}(t)+w_{\mathrm{u}} u^{2}(t)\right] \mathrm{d} t
$$

where $\boldsymbol{x}(t)$ is the state vector, $u(t)$ is the input of the process, respectively. The positive definite $\boldsymbol{W}_{\mathrm{x}}$ stands for penalizing the variations in the state space, $w_{\mathrm{u}}$ is for penalizing the energy of the control action. The classical solution, minimizing (55) is a negative state feedback $(S F)$ 


$$
u(t)=-\boldsymbol{k}_{\mathrm{LQ}}^{\mathrm{T}} \boldsymbol{x}(t)
$$

where $\boldsymbol{k}_{\mathrm{LQ}}^{\mathrm{T}}$ is given by

$$
\boldsymbol{k}_{\mathrm{LQ}}^{\mathrm{T}}=\frac{1}{w_{\mathrm{u}}} \boldsymbol{b}^{\mathrm{T}} \boldsymbol{P}
$$

The symmetric positive semi definite matrix $\boldsymbol{P}$ can be obtained from the solution of the algebraic RICCATI equation [4]

$$
\boldsymbol{P} \boldsymbol{A}+\boldsymbol{A}^{\mathrm{T}} \boldsymbol{P}-\frac{1}{w_{\mathrm{u}}} \boldsymbol{P} \boldsymbol{b} \boldsymbol{b}^{\mathrm{T}} \boldsymbol{P}=-\boldsymbol{W}_{\mathrm{x}}
$$

Analytic solution is not possible, because this equation is nonlinear in $\boldsymbol{P}$, therefore only numeric solution can be obtained by MATLAB and other CACSD programs.

Introducing the orthogonal factorization

$$
\boldsymbol{W}_{\mathrm{x}}=\boldsymbol{G}^{\mathrm{T}} \boldsymbol{G}
$$

the closed-loop system is stable if the auxiliary process

$$
\boldsymbol{v}=\boldsymbol{G} \boldsymbol{x}
$$

is observable.

The Frequency Domain Solution of the LQR Problem

The $L Q R$ approach is widely used for control problems in all over the world, however, in a practical problem it is not an easy task to find the best $\boldsymbol{W}_{\mathrm{x}}$ and $w_{\mathrm{u}}$ weights, which are usually obtained by trial and error iterative methods. The $L Q R$ problem has an almost forgotten frequency domain solution, too, which will give us a deterministic design process to find useful relationships between the classical pole placement $S F$ solution and the $L Q R$ paradigm. It can be shown that the simpler dyadic factorization [7]

$$
\boldsymbol{W}_{\mathrm{x}}=\boldsymbol{g} \boldsymbol{g}^{\mathrm{T}} ; \boldsymbol{g}=\left[g_{1}, \ldots, g_{n-1}, g_{n}\right]^{\mathrm{T}}
$$

can also be used. The frequency domain condition of the minimum of (55) is called the KALMAN equation [7], [8] or sometimes it is named frequency domain identity

$$
w_{\mathrm{u}}\left|1+\boldsymbol{k}^{\mathrm{T}} \boldsymbol{\Phi}(s) \boldsymbol{b}\right|^{2}=w_{\mathrm{u}}+\left|\boldsymbol{g}^{\mathrm{T}} \boldsymbol{\Phi}(s) \boldsymbol{b}\right|^{2}
$$

Assuming unity weight $w_{\mathrm{u}} \equiv 1$ the equation becomes even simpler

$$
\left|1+\boldsymbol{k}^{\mathrm{T}} \boldsymbol{\Phi}(s) \boldsymbol{b}\right|^{2}=1+\left\|\boldsymbol{g}^{\mathrm{T}} \boldsymbol{\Phi}(s) \boldsymbol{b}\right\|_{2}^{2}=1+\left|\boldsymbol{g}^{\mathrm{T}} \boldsymbol{\Phi}(s) \boldsymbol{b}\right|^{2}
$$

Using the well known relationship of complex functions

$$
\{Z(s)\}^{2}=|Z(s)|^{2}=Z(s) Z(-s)
$$

and introducing the $(n-1)$ th order polynomial $\mathbb{G}(s)$ as the numerator of

$$
H(s)=\boldsymbol{g}^{\mathrm{T}} \boldsymbol{\Phi}(s) \boldsymbol{b}=\frac{\mathbb{G}(s)}{\mathbb{A}(s)}=\frac{g_{1} s^{n-1}+\ldots+g_{n-1} s+g_{n}}{\mathbb{A}(s)}(6
$$

the equation (63) can be rearranged into a new form

$$
\begin{gathered}
\mathbb{R}(s) \mathbb{R}(-s)=\left[\mathbb{A}(s)+\boldsymbol{k}^{\mathrm{T}} \boldsymbol{\Psi}(s) \boldsymbol{b}\right]\left[\mathbb{A}(-s)+\boldsymbol{k}^{\mathrm{T}} \boldsymbol{\Psi}(-s) \boldsymbol{b}\right]= \\
=\mathbb{A}(s) \mathbb{A}(-s)+\underbrace{\left[\boldsymbol{g}^{\mathrm{T}} \boldsymbol{\Psi}(s) \boldsymbol{b}\right]}_{\mathbb{G}(s)} \underbrace{\left[\boldsymbol{g}^{\mathrm{T}} \boldsymbol{\Psi}(-s) \boldsymbol{b}\right]}_{\mathbb{G}(-s)}
\end{gathered}
$$

which provides the quadratic polynomial solution of the KALMAN equation. Thus the final quadratic equation, ensuring relationship between the process $\mathbb{A}(s)$, design $\mathbb{R}(s)$ and weighting $\mathbb{G}(s)$ polynomials, is

$$
\begin{aligned}
& \mathbb{R}(s) \mathbb{R}(-s)=\mathbb{A}(s) \mathbb{A}(-s)+\mathbb{G}(s) \mathbb{G}(-s) \\
& \text { or }|\mathbb{R}(s)|^{2}=|\mathbb{A}(s)|^{2}+|\mathbb{G}(s)|^{2}
\end{aligned}
$$

or in the general form

$$
w_{\mathrm{u}}|\mathbb{R}(s)|^{2}=w_{\mathrm{u}}|\mathbb{A}(s)|^{2}+|\mathbb{G}(s)|^{2}
$$

Observe that the solution tends to $\mathbb{R}(s)=\mathbb{A}(s)$ if $w_{\mathrm{u}} \rightarrow \infty$ and $\boldsymbol{g}^{\mathrm{T}} \boldsymbol{x}=0$ if $w_{\mathrm{u}} \rightarrow 0$. Do not forget that $\mathbb{K}(s)$ and $\mathbb{G}(s)$ are $(n-1)$ th order [8].

Some Anomalies in the LQR Problem

The solution of the polynomial equation can be a direct coefficient comparison or a spectral factorization approach [8]. Consider some examples in the sequel.

\section{Example 1}

Consider a first order example with

$$
\mathbb{A}(s)=s+a_{1} ; \mathbb{R}(s)=s+r_{1} \text { thus } \mathbb{G}(s)=g_{1}
$$

The two sides of (67) are

$$
-s^{2}-r_{1} s+r_{1} s+r_{1}^{2}=-s^{2}-a_{1} s+a_{1} s+a_{1}^{2}+g_{1}^{2}
$$

and the solution is

$$
r_{1}^{2}=a_{1}^{2}+g_{1}^{2}>0
$$

and

$$
k_{1}=r_{1}-a_{1}=\sqrt{a_{1}^{2}+g_{1}^{2}}-a_{1}>0
$$

If we want to ensure (place) a required pole then the necessary weight in the $L Q R$ problem is

$$
g_{1}=\sqrt{r_{1}^{2}-a_{1}^{2}}
$$

It is easy to see that only such $r_{1}$ can be placed, which fulfills the condition 


$$
r_{1}^{2}>a_{1}^{2} \Rightarrow\left|r_{1}\right|>\left|a_{1}\right| \Rightarrow r_{1}>\left|a_{1}\right|
$$

for stable design polynomial $\mathbb{R}(s)$.

So this example shows that only a faster pole can be placed by the $L Q R$ optimization comparing to the original process pole.

\section{Example 2}

Consider a second order example with

$$
\begin{aligned}
& \mathbb{A}(s)=s^{2}+a_{1} s+a_{2} \quad ; \quad \mathbb{R}(s)=s^{2}+r_{1} s+r_{2} \\
& \text { thus } \quad \mathbb{G}(s)=g_{1} s+g_{2}
\end{aligned}
$$

The two sides of (67) are now

$$
\begin{aligned}
& \left(s^{2}+r_{1} s+r_{2}\right)\left(s^{2}-r_{1} s+r_{2}\right)=\left(s^{2}+a_{1} s+a_{2}\right) \\
& \left(s^{2}-a_{1} s+a_{2}\right)+\left(g_{1} s+g_{2}\right)\left(-g_{1} s+g_{2}\right)
\end{aligned}
$$

and the solutions are

$$
\begin{gathered}
r_{2}=\sqrt{a_{2}^{2}+g_{2}^{2}}>a_{2} \\
r_{1}=\sqrt{2\left(\sqrt{a_{2}^{2}+g_{2}^{2}}-a_{2}\right)+\left(a_{1}^{2}+g_{1}^{2}\right)}= \\
=\sqrt{2\left(r_{2}-a_{2}\right)+\left(a_{1}^{2}+g_{1}^{2}\right)}>a_{1}>0
\end{gathered}
$$

The $S F$ to be applied is given by

$$
k_{1}=r_{1}-a_{1}>0 ; k_{2}=r_{2}-a_{2}>0
$$

For pole placement the necessary $L Q R$ weights are

$$
g_{2}=\sqrt{r_{2}^{2}-a_{2}^{2}}
$$

and

$$
g_{1}=\sqrt{r_{2}^{2}-a_{1}^{2}-2\left(r_{2}-a_{2}\right)}=\sqrt{2\left(\mu_{\mathrm{r}}^{2}-\mu_{\mathrm{a}}^{2}\right)}
$$

where

$$
\mu_{\mathrm{r}}^{2}=\frac{r_{1}^{2}-2 r_{2}}{2}=\frac{\left(s_{1}^{\mathrm{r}}\right)^{2}+\left(s_{2}^{\mathrm{r}}\right)^{2}}{2}
$$

and

$$
\mu_{\mathrm{a}}^{2}=\frac{a_{1}^{2}-2 a_{2}}{2}=\frac{\left(s_{1}^{\mathrm{a}}\right)^{2}+\left(s_{2}^{\mathrm{a}}\right)^{2}}{2}
$$

It is easy to see that there are such $\left\{r_{1}, r_{2}\right\}$ domains, which can not be reached by any $\left\{g_{1}, g_{2}\right\}$ selection !!! These conditions are

$$
r_{2}^{2}>a_{2}^{2} \Rightarrow\left|r_{2}\right|>\left|a_{2}\right| \Rightarrow r_{2}>\left|a_{2}\right|
$$

and

$$
r_{2} \geq \frac{r_{1}^{2}}{2}-2 \mu_{\mathrm{a}}^{2}=\frac{r_{1}^{2}}{2}-\left(a_{1}^{2}-2 a_{2}\right)
$$

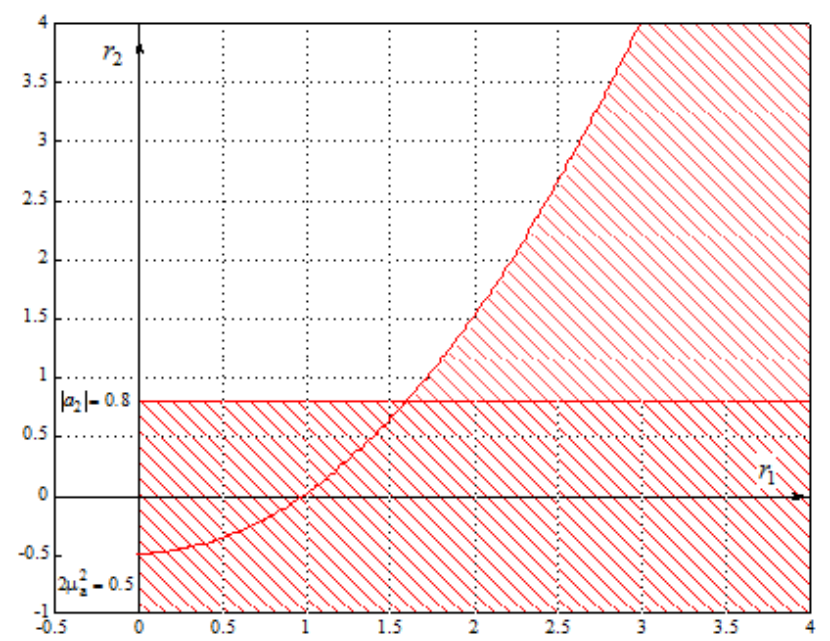

Figure 19. Unreachable design parameter domains

These conditions are graphically demonstrated on Fig.19, where the shaded area shows the unreachable design parameters for the case of open-loop process parameters $\left|a_{2}\right|=0.8$ and $2 \mu_{\mathrm{a}}^{2}=0.5$.

One can check these results either via the solution of the RICCATI equation (very time consuming method) or by the spectral factorization approach

$$
\begin{aligned}
\mathbb{R}(s) \mathbb{R}(-s)= & {[\mathbb{A}(s) \mathbb{A}(-s)+\mathbb{G}(-s) \mathbb{G}(s)]^{+} } \\
& {[\mathbb{A}(s) \mathbb{A}(-s)+\mathbb{G}(-s) \mathbb{G}(s)]^{-} }
\end{aligned}
$$

as the solution of (67), i.e., by

$$
\mathbb{R}(s)=[\mathbb{A}(s) \mathbb{A}(-s)+\mathbb{G}(s) \mathbb{G}(-s)]^{+}
$$

Solutions for LQR-pole placement

A new criterion

$$
I=\frac{1}{2} \int_{0}^{\infty}\left[\boldsymbol{x}^{\mathrm{T}}(t) \boldsymbol{W}_{\mathrm{x}} \boldsymbol{x}(t)+\boldsymbol{x}^{\mathrm{T}}(t) \boldsymbol{w}_{\mathrm{xu}} u(t)+W_{\mathrm{u}} u^{2}(t)\right] \mathrm{d} t
$$

was proposed by Bokor and Keviczky, which solves the problem and offers a possible way to overcome this anomaly of the standard method.

The full pole-placement solution can be obtained only if the criterion (86) is used, which penalizes interaction of the state vector and the control action by the $\boldsymbol{x}^{\mathrm{T}}(t) \boldsymbol{w}_{\mathrm{xu}} u(t)$ cross-term. The optimal state feedback can be provided by

$$
\boldsymbol{k}=-w_{\mathrm{u}}^{-1}\left(\boldsymbol{w}_{\mathrm{xu}}+\boldsymbol{P} \boldsymbol{b}\right) \text { or } \boldsymbol{k}^{\mathrm{T}}=-w_{\mathrm{u}}^{-1}\left(\boldsymbol{w}_{\mathrm{ux}}+\boldsymbol{b}^{\mathrm{T}} \boldsymbol{P}\right)
$$

introduced in [11] and [12]. 


\section{Conclusions}

The purpose of this paper is to highlight some interesting, may be philosophical, paradigm of modeling and control as the extended abstract indicated.

Such special and not widely known problems are discussed here, which are worth further study and investigation.

"I believe that the progress of science should be rather measured by the raised and not by the solved problems !" as Eddington stated !!!

\section{REFERENCES}

[1] L. Keviczky, Cs. Bányász. Two-Degree-of-Freedom Control Systems (The Youla Parameterization Approach), Elsevier, Academic Press, 2015.

[2] L. Keviczky, Cs. Bányász. Generic two-degree of freedom control systems for linear and nonlinear processes, J. Systems Science, Vol. 26, No. 4, 5-24, 2001.

[3] L. Ljung. System Identification, Theory for the User, Second Edition, Prentice Hall, 1999.

[4] J.M. Maciejowski. Multivariable Feedback Design, Addison Wesley, 1989.
[5] D.C. Youla, J.J. Bongiorno, C. N. Lu. Single-loop feedback stabilization of linear multivariable dynamical plants, Automatica, Vol. 10, No. 2, 159-173, 1974.

[6] R. Kalman. Prejudice free modeling and identification, (Plenary Paper), SYSID’91, Budapest, 1991.

[7] F. Csáki. State-Space Methods for Control Systems, Akadémiai Kiadó, Budapest, 1978.

[8] T. Kailath. Linear Systems, Prentice Hall, 1980.

[9] Cs. Bányász, L. Keviczky. State-feedback solutions via transfer function representations, J. Systems Science, Vol. 30, No. 2, 21-34, 2004.

[10] L. Keviczky, Cs. Bányász. Model error properties of observer-based state-feedback controller, 6th Int. Conf. System Identification and Control Problems SICPRO'07, Moscow, 879-888, 2007.

[11] C.D. Johnson. The unreachable poles in LQR theory: analysis and remedy, Int. J. Control, Vol. 47, No. 3, 697-709, 1988.

[12] L. Keviczky. J. Bokor. On the solution of an LQ control problem anomaly, Proc. of the Automation and Applied Computer Science Workshop AACS'11 (50th Anniversary of the Dept. of Automation and Applied Informatics), Budapest, 22-37, 2011.

[13] L. Keviczky, J. Bokor. On an LQ problem anomaly and a possible solution, 14th IASTED Int. Conf. on Control and Applications, Crete, 781-013, 2012. 